\title{
SEQUENTIAL FIXED-WIDTH CONFIDENCE INTERVAL ESTIMATION FOR THE PERCENTILES OF A NORMAL DISTRIBUTION
}

\author{
Keiichi Hirose* and Eiichi Isogai**
}

\begin{abstract}
In this paper we consider sequential fixed-width confidence interval estimation for the percentiles of a normal distribution with unknown mean and variance. We construct two kinds of confidence intervals by using a stopping rule and show their asymptotic consistency. We also try to compare the performance of these confidence intervals by the simulation results.
\end{abstract}

Key words: Asymptotic consistency, Bias correction, Second-order expansion, Expected sample size, Stopping rule

\section{Introduction}

Let $X_{1}, X_{2}, \cdots$ be independent and identically distributed normal random variables with unknown mean $\mu \in(-\infty, \infty)$ and variance $\sigma^{2} \in(0, \infty)$. We want to estimate $\theta=a \mu+b \sigma$ with given constants $a$ and $b$, including the $p$-th percentiles.

In this paper, we will construct sequential confidence intervals of $\theta$ with preassigned fixed-width $2 d$ and coverage probability $1-\alpha$. For a parameter of a negative exponential distribution, Isogai et al. (1995) and Hirose et al. (1997) have considered this problem. The problem of sequential fixed-width confidence interval estimation for the mean has been studied by Chow and Robbins (1965), Woodroofe (1977), Callaert and Janssen (1981), Jurečková (1991) and Mukhopadhyay and Datta (1996), among others. Ghosh and Mukhopadhyay (1989) considered the problem of minimum risk point estimation of $\theta$. We are not aware, however, of the problem of the confidence interval estimation for $\theta$.

The aim of this paper is to construct sequential confidence intervals of $\theta$. In Section 2 we will propose two kinds of sequential confidence intervals, corresponding to the natural sequential estimator of $\theta$ and its bias-corrected version, respectively, and show their asymptotic consistency as $d$ approaches zero. We will also derive the second order asymptotic expansion of the expected sample size. Section 3 gives simulation results to compare the performance of these confidence intervals. The proofs of the results in this paper will be presented in Section 4 .

Received March, 1997. Revised June, 1997. Accepted September, 1997.

* Department of Mathematical Science, Graduate School of Science and Technology, Niigata University, Niigata, 950-21, Japan [hirose@ dmis.gs.niigata-u.ac.jp]

* Department of Mathematics, Faculty of Science, Niigata University, Niigata 950-21, Japan [isogai@math.sc.niigata-u.ac.jp] 


\section{Fixed-width confidence intervals}

In this section we propose a stopping rule in order to stop sampling and construct sequential confidence intervals of $\theta=a \mu+b \sigma$ with preassigned fixedwidth $2 d$ and coverage probability $1-\alpha$ by using the stopping rule. Then we show their asymptotic consistency as $d$ tends to zero. We also derive the second order asymptotic expansion of the expected sample size.

Let $d>0$ and $\alpha \in(0,1)$ be given. We choose the constant $z=z_{\alpha}>0$ such that $\Phi(z)-\Phi(-z)=1-\alpha$ where $\Phi$ denotes the standard normal distribution function. For $n \geq 2$ set

$$
\begin{aligned}
& \bar{X}_{n}=\frac{1}{n} \sum_{i=1}^{n} X_{i}, \quad s_{n}^{2}=\frac{1}{n-1} \sum_{i=1}^{n}\left(X_{i}-\bar{X}_{n}\right)^{2}, \\
& \hat{\theta}_{n}=a \bar{X}_{n}+b s_{n} \quad \text { and } \quad I_{n}=I_{n}(d)=\left[\hat{\theta}_{n}-d, \hat{\theta}_{n}+d\right] .
\end{aligned}
$$

Throughout this paper we assume

$$
m \equiv 2 a^{2}+b^{2}>0 .
$$

We will now derive the asymptotic expression of the probability $P\left\{\theta \in I_{n}\right\}$ for large $n$. For $n \geq 1$ put

$$
Y_{n}=\frac{1}{n(n+1) \sigma^{2}}\left(\sum_{i=1}^{n}\left(X_{i}-X_{n+1}\right)\right)^{2} \quad \text { and } \quad \bar{Y}_{n}=\frac{1}{n} \sum_{i=1}^{n} Y_{i} .
$$

Then it is well known that $Y_{1}, Y_{2}, \cdots$ are independent and identically distributed random variables having a chi-square distribution with one degree of freedom and that

$$
s_{n}^{2}=\sigma^{2} \bar{Y}_{n-1} .
$$

For $n \geq 2$ set

$$
\begin{aligned}
& U_{n}=\frac{\sqrt{n}\left(\bar{X}_{n}-\mu\right)}{\sigma}, \quad V_{n}=\frac{\sqrt{n-1}\left(\bar{Y}_{n-1}-1\right)}{\sqrt{2}}, \\
& W_{n}={ }^{T}\left(U_{n}, V_{n}\right) \text { and } c={ }^{r}\left(c_{1}, c_{2}\right) \text { for any real numbers } c_{1} \text { and } c_{2},
\end{aligned}
$$

where $T$ denotes transpose. Then it follows from (2.3) and (2.4) that

$$
\sqrt{n}\left(\hat{\theta}_{n}-\theta\right)=a \sigma U_{n}+\sqrt{\frac{2 n}{n-1}} \cdot \frac{b \sigma^{2}}{s_{n}+\sigma} V_{n}
$$

and $U_{n}$ and $V_{n}$ are independent because of the independency of $\bar{X}_{n}$ and $s_{n}^{2}$. For any real numbers $c_{1}, c_{2}$ and $t$, by a central limit theorem and the independency of $U_{n}$ and $V_{n}$ we have with $i^{2}=-1$

$$
E\left[\exp \left(i t^{T} c W_{n}\right)\right] \rightarrow E\left[\exp \left(i t^{T} c W\right)\right] \text { as } n \rightarrow \infty,
$$

which implies

$$
{ }^{T} C W_{n} \stackrel{L}{\longrightarrow}{ }^{T} C W \text { as } n \rightarrow \infty,
$$

where " $L$ " stands for convergence in law and $W={ }^{T}(U, V)$ denotes a twodimensional normal random vector with mean vector ${ }^{T}(0,0)$ and covariance 
matrix $I$ (the unit matrix). Thus by the Cramér-Wold device (see Billingsley (1968), p. 49) we get

$$
W_{n} \stackrel{L}{\longrightarrow} W \text { as } n \rightarrow \infty
$$

which yields

$$
a \sigma U_{n}+\frac{b \sigma}{\sqrt{2}} V_{n} \stackrel{L}{\longrightarrow} Z \text { as } n \rightarrow \infty
$$

where $Z \equiv a \sigma U+(b \sigma / \sqrt{2}) V$ has a normal distribution with mean 0 and variance $m \sigma^{2} / 2$. Since $s_{n}^{2} \stackrel{u s}{\longrightarrow} \sigma^{2}$ as $n \rightarrow \infty$, we have by (2.6)

$$
\left|\sqrt{\frac{2 n}{n-1}} \cdot \frac{b \sigma^{2}}{s_{n}+\sigma} V_{n}-\frac{b \sigma}{\sqrt{2}} V_{n}\right| \stackrel{p}{\longrightarrow} 0 \text { as } n \rightarrow \infty
$$

where " $\stackrel{a . .}{\longrightarrow}$ " and “ $\longrightarrow$ " denote almost sure convergence and convergence in probability, respectively. Hence it follows from (2.5) and (2.7) that $\sqrt{n}\left(\hat{\theta}_{n}-\theta\right)$ $\stackrel{L}{\longrightarrow} Z$ as $n \rightarrow \infty$, which, together with $(2.5)$, yields

$$
\sqrt{\frac{2 n}{m \sigma^{2}}}\left(\hat{\theta}_{n}-\theta\right) \stackrel{L}{\longrightarrow} N(0,1) \text { as } n \rightarrow \infty
$$

where $N(0, \beta)$ denotes a normal random variable with mean 0 and variance $\beta$. Therefore

$$
\begin{aligned}
P\left\{\theta \in I_{n}\right\} & =P\left\{\left|\sqrt{\frac{2 n}{m \sigma^{2}}}\left(\hat{\theta}_{n}-\theta\right)\right| \leq \sqrt{\frac{2 n}{m \sigma^{2}}} d\right\} \\
& \sim \Phi\left(\sqrt{\frac{2 n}{m \sigma^{2}}} d\right)-\Phi\left(-\sqrt{\frac{2 n}{m \sigma^{2}}} d\right) \text { as } n \rightarrow \infty
\end{aligned}
$$

Now for large $n$

$$
\sqrt{\frac{2 n}{m \sigma^{2}}} d \geq z \text { or equivalently } n \geq \frac{m z^{2}}{2 d^{2}} \sigma^{2}
$$

implies that $P\left\{\theta \in I_{n}\right\} \geq 1-\alpha$. Let

$$
n^{*}=\frac{m z^{2}}{2 d^{2}} \sigma^{2}
$$

Here, for simplicity we assume that $n^{*}$ is a positive integer. Clearly $n^{*}$ is the asymptotic optimal fixed sample size. Since $\sigma^{2}$ is unknown, so is $n^{*}$. Hence there is no fixed sample size procedure. Thus we propose the following stopping rule:

$$
N=N_{d}(L)=\inf \left\{n \geq k: n \geq \frac{m z^{2}}{2 d^{2}} s_{n}^{2} L_{n}\right\},
$$

where $k \geq 2$ is the starting sample size, $L_{x}$ is a positive continuous function on $(0, \infty)$ for which 


$$
L_{x}=1+L x^{-1}+o\left(x^{-1}\right) \text { as } x \rightarrow \infty \text { with a constant } L .
$$

REMARK 2.1. Woodroofe (1977) had considered this problem with $a=1$ and $b=0$. In this case we get $m=2$ in (2.1). Then the stopping rule $N$ in (2.9) with $m=2$ and $L=2 \Delta$ is the same as in (4.3) of Woodroofe (1977).

REMARK 2.2. By Lemma 1 of Chow and Robbins (1965) we have

$$
\begin{aligned}
& P\{N<\infty\}=1 \text { for all } d>0, \\
& N \stackrel{a . s .}{\longrightarrow} \infty \text { and } N / n^{*} \stackrel{u . .}{\longrightarrow} 1 \text { as } d \rightarrow 0 .
\end{aligned}
$$

Before providing the results, we note that all the proofs of the results in this section will be provided in the final section. Now, the natural sequential estimator of $\theta$ is given by

$$
\hat{\theta}_{N}^{(1)}=a \bar{X}_{N}+b s_{N} .
$$

First, we shall evaluate the bias of the sequential estimator $\hat{\theta}_{N}^{(1)}$. The following lemma presents the bias of the estimator $s_{N}$ of $\sigma$.

LEMMA 2.1. If $k>7$ in (2.9) then

$$
E\left(s_{N}\right)-\sigma=-\frac{5 \sigma}{4}\left(n^{*}\right)^{-1}+o\left(d^{2}\right) \text { as } d \rightarrow 0 .
$$

From this lemma we get the bias of $\hat{\theta}_{N}^{(1)}$.

PROPOSITION 2.1. If $k>7$ then

$$
E\left(\hat{\theta}_{N}^{(1)}\right)-\theta=-\frac{5 b \sigma}{4}\left(n^{*}\right)^{-1}+o\left(d^{2}\right) \quad \text { as } d \rightarrow 0 .
$$

Taking Proposition 2.1 into account, we consider the following bias-corrected sequential estimator $\hat{\theta}_{N}^{(2)}$ of $\theta$ :

$$
\hat{\theta}_{N}^{(2)}=\hat{\theta}_{N}^{(1)}+\frac{5 b d^{2}}{2 m z^{2}} s_{N}^{-1} .
$$

We may, of course, be able to consider other bias-corrected sequential estimators. Then the bias of $\hat{\theta}_{N}^{(2)}$ is given by

PROPOSITION 2.2. If $k>7$ then

$$
E\left(\hat{\theta}_{N}^{(2)}\right)-\theta=o\left(d^{2}\right) \quad \text { as } d \rightarrow 0 .
$$

We shall now construct the confidence intervals for $\theta$. For $j=1,2$ let

$$
I_{N}^{(j)}=I_{N}^{(j)}(d, L)=\left[\hat{\theta}_{N}^{(j)}-d, \hat{\theta}_{N}^{(j)}+d\right] .
$$

Once the sampling stops, $I_{N}^{(j)}(j=1,2)$ is used as the confidence interval of $\theta$ with width $2 d$ and coverage probability $1-\alpha$. The asymptotic consistency of $I_{N}^{(j)}(j=1,2)$ in the sense of Chow and Robbins (1965) can then be shown by the following theorem. 
THEOREM 2.1. If $k>1$ in (2.9) for $j=1$ and $k>7$ for $j=2$ then

$$
\lim _{d \rightarrow 0} P\left\{\theta \in I_{N}^{(j)}\right\}=1-\alpha \quad \text { for } \quad j=1,2 .
$$

Finally we provide the second-order asymptotic expansion of the expected sample size.

THEOREM 2.2. If $k>3$ then

$$
E(N)=n^{*}+\nu+L-2+o(1) \text { as } d \rightarrow 0 \text {, }
$$

where $\nu$ is the constant given in Lemma 4.1 (ii) of Section 4.

REMARK 2.3. This result with $L=2 \Delta$ is the same as in (4.3) of Woodroofe (1977).

\section{Simulation results}

In this section we present the simulation results for two sequential confidence intervals given in Section 2. Throughout the simulations we consider $\mu=0, \sigma^{2}=1, L_{x}=1+1.18 x^{-1}+o\left(x^{-1}\right)$ in (2.10), $k=10$ in $(2.9), n^{*}=50,100$, 500,1000 in (2.8), and $\alpha=0.05$, that is, the sequential $95 \%$ confidence intervals of $\theta=a \mu+b \sigma$. We note that $L=1.18$ in Theorem 2.2 is chosen in order to adjust the expected sample size $E(N)$ to the asymptotic optimal fixed sample size $n^{*}$. For $d>0$ put

$$
\theta_{j}=E\left(\hat{\theta}_{N}^{(j)}\right) \quad \text { and } \quad p_{j}=P\left\{\theta \in I_{N}^{(j)}\right\} \quad \text { for } j=1,2 .
$$

All $\theta_{j}$ and $p_{j}(j=1,2)$ in the tables below were estimated by repeating the experiment 20,000 times. Table 1,2 and 3 show the results for $(a, b)=$ $(1,0.67452),(1,2)$ and $(1,3)$, respectively.

From the simulation results we feel that the bias of the estimator $\hat{\theta}_{N}^{(1)}$ of $\theta$ is corrected. However, it appears that the coverage probabilities of the sequential confidence intervals $I_{N}^{(2)}$ constructed by using $\hat{\theta}_{N}^{(2)}$ is not always higher than that of $I_{N}^{(1)}$ by $\hat{\theta}_{N}^{(1)}$. It is therefore interesting to compare the performance of these confidence intervals by evaluating their coverage probabilities more precisely. Furthermore, we are interested in investigating the mean square error (m.s.e) and the variance for the estimators $\hat{\theta}_{N}^{(1)}$ and $\hat{\theta}_{N}^{(2)}$ of $\theta$. The

Table 1. $\theta=\mu+0.67452 \sigma$ (the $75 \%$ point)

\begin{tabular}{c|cccc}
\hline \multirow{2}{*}{} & \multicolumn{4}{|c}{$n^{*}$} \\
\cline { 2 - 5 } & 50 & 100 & 500 & 1000 \\
\hline$\theta$ & 0.67452 & 0.67452 & 0.67452 & 0.67452 \\
$d$ & 0.307100 & 0.217152 & 0.097114 & 0.068670 \\
\hline$E(N)$ & 52.0393 & 101.4368 & 499.1302 & 1002.7206 \\
\hline$\theta_{1}$ & 0.652689 & 0.673278 & 0.668962 & 0.676934 \\
$\theta_{2}$ & 0.670139 & 0.681779 & 0.670655 & 0.677778 \\
\hline$p_{1}$ & 0.84865 & 0.96585 & 0.94030 & 0.94200 \\
$p_{2}$ & 0.84865 & 0.96585 & 0.95750 & 0.93675 \\
\hline
\end{tabular}


Table 2. $\theta=\mu+2 \sigma$

\begin{tabular}{c|cccc}
\hline \multirow{2}{*}{} & \multicolumn{4}{|c}{$n^{*}$} \\
\cline { 2 - 5 } & 50 & 100 & 500 & 1000 \\
\hline$\theta$ & 2.0 & 2.0 & 2.0 & 2.0 \\
$d$ & 0.480100 & 0.339482 & 0.151821 & 0.107354 \\
\hline$E(N)$ & 46.8588 & 101.0119 & 500.8920 & 1003.7228 \\
\hline$\theta_{1}$ & 1.878739 & 1.981119 & 1.991994 & 2.003431 \\
$\theta_{2}$ & 1.932594 & 2.006457 & 1.997006 & 2.005931 \\
\hline$p_{1}$ & 0.84100 & 0.94325 & 0.96615 & 0.95090 \\
$p_{2}$ & 0.84100 & 0.94725 & 0.96615 & 0.95850 \\
\hline
\end{tabular}

Table 3. $\theta=\mu+3 \sigma$

\begin{tabular}{c|cccc}
\hline \multirow{2}{*}{} & \multicolumn{4}{|c}{$n^{*}$} \\
\cline { 2 - 5 } & 50 & 100 & 500 & 1000 \\
\hline$\theta$ & 3.0 & 3.0 & 3.0 & 3.0 \\
$d$ & 0.650058 & 0.459661 & 0.205567 & 0.145357 \\
\hline$E(N)$ & 51.0504 & 99.0922 & 498.2734 & 1001.9756 \\
\hline$\theta_{1}$ & 2.975254 & 2.940844 & 2.986245 & 3.002163 \\
$\theta_{2}$ & 3.053375 & 2.979244 & 2.993785 & 3.005915 \\
\hline$p_{1}$ & 0.91880 & 0.91085 & 0.95215 & 0.96315 \\
$p_{2}$ & 0.91880 & 0.93080 & 0.96085 & 0.96315 \\
\hline
\end{tabular}

following results give the m.s.e and the variance of the estimators for $\theta$. The proof of the results in this section will be briefly given in Section 4 .

THEOREM 3.1. If $k>16$ then as $d \rightarrow 0$

$$
E\left\{\hat{\theta}_{N}^{(1)}-\theta\right\}^{2}=\frac{m \sigma^{2}}{2 n^{*}}+\left((-\nu-L+4) \frac{m}{2}+\frac{87}{16} b^{2}\right) \frac{\sigma^{2}}{\left(n^{*}\right)^{2}}+o\left(d^{4}\right)
$$

and

$$
E\left\{\hat{\theta}_{N}^{(2)}-\theta\right\}^{2}=\frac{m \sigma^{2}}{2 n^{*}}+\left((-\nu-L+4) \frac{m}{2}+\frac{21}{8} b^{2}\right) \frac{\sigma^{2}}{\left(n^{*}\right)^{2}}+o\left(d^{4}\right) .
$$

From Theorem 3.1 and Propositions 2.1 and 2.2 we readily get the variance of the estimators $\hat{\theta}_{N}^{(1)}$ and $\hat{\theta}_{N}^{(2)}$ of $\theta$.

PROPOSITION 3.1. If $k>16$ then as $d \rightarrow 0$

$$
\operatorname{Var}\left(\hat{\theta}_{N}^{(1)}\right)=\frac{m \sigma^{2}}{2 n^{*}}+\left((-\nu-L+4) \frac{m}{2}+\frac{31}{8} b^{2}\right) \frac{\sigma^{2}}{\left(n^{*}\right)^{2}}+o\left(d^{4}\right)
$$

and

$$
\operatorname{Var}\left(\hat{\theta}_{N}^{(2)}\right)=\frac{m \sigma^{2}}{2 n^{*}}+\left((-\nu-L+4) \frac{m}{2}+\frac{21}{8} b^{2}\right) \frac{\sigma^{2}}{\left(n^{*}\right)^{2}}+o\left(d^{4}\right) .
$$

REMARK 3.1. Since the results of Theorem 3.1 and Proposition 3.1, indicate that both the m.s.e and the variance of the bias-corrected estimator $\hat{\theta}_{N}^{(2)}$ are 
a little bit better than those of the natural estimator $\hat{\theta}_{N}^{(1)}$, it seems that the bias-correction is efficient in the case of sequential point estimation for $\theta$. However, it appears that the variances are much larger with respect to the bias of the estimators $\hat{\theta}_{N}^{(1)}$ and $\hat{\theta}_{N}^{(2)}$. Therefore we feel that this fact justifies the simulation results.

\section{Proofs}

In this section all the proofs of the results in Sections 2 and 3 are given. By (2.2), (2.3), (2.8) and (2.9) we get

$$
N=\inf \left\{n \geq k: \sum_{i=1}^{n-1} Y_{i} \leq\left(n^{*}\right)^{-1}(n-1)^{2} l_{n-1}\right\}
$$

where $Y_{n}(n=1,2, \cdots)$ are given in $(2.2), l_{x}=1+l x^{-1}+o\left(x^{-1}\right)$ as $x \rightarrow \infty$ with the constant $l=1-L$ and $L$ is the constant given in (2.10). Let

$$
t=t_{d}(l)=\inf \left\{n \geq k-1: \sum_{i=1}^{n} Y_{i} \leq\left(n^{*}\right)^{-1} n^{2} l_{n}\right\} .
$$

Then

$$
N=t+1 \text { and } s_{N}^{2}=\sigma^{2} \bar{Y}_{t} .
$$

We introduce notation used in this section. Let

$$
\begin{aligned}
& t^{*}=\left(n^{*}\right)^{-1 / 2}\left(t-n^{*}\right), \quad S_{n}=\sum_{i=1}^{n} Y_{i}, \quad R=S_{t}-t, \quad T=\left(n^{*}\right)^{-1 / 2} R, \\
& \eta=t / n^{*}, \quad r=\left(n^{*}\right)^{-1} t^{2} l_{t}-S_{t}=\eta t l_{t}-S_{t}
\end{aligned}
$$

and let $\chi_{n}^{2}$ denote a random variable having a chi-square distribution with $n$ degrees of freedom. Throughout the remainder of this paper, we consider the case where $d \rightarrow 0$ and let $d_{0}>0$ be sufficiently small. Before proving the results in Section 2, we give four lemmas. From (4.1), Theorems 2.1-2.4 and Lemmas 2.1 and 2.3 of Woodroofe (1977) we have

LEMMA 4.1. The following facts hold.

(i) $\quad t \stackrel{\text { a.s. }}{\longrightarrow} \infty, \quad \eta \stackrel{\text { a.s. }}{\longrightarrow} 1$ and $\bar{Y}_{t} \stackrel{\text { a.s. }}{\longrightarrow} 1$.

(ii) $\quad t^{*} \stackrel{L}{\longrightarrow} N(0,2), \quad r \stackrel{L}{\longrightarrow} H$

and $t^{*}$ and $r$ are asymptotically independent where $H$ is the random variable given in Theorem 2.1 of Woodroofe (1977),

$$
\begin{aligned}
\nu & =E(H)=\frac{3}{2}-\sum_{n=1}^{\infty} n^{-1} E\left\{\max \left(0, S_{n}-2 n\right)\right\} \\
& \fallingdotseq 0.820 \text { by Table } 2.1 \text { of Woodroofe (1977). }
\end{aligned}
$$

(iii) For any fixed $s>0, E\left(\eta^{s}\right)=O(1)$ and $E\left(r^{s}\right)=O(1)$.

(iv) $P\left(\eta \leq \frac{1}{2}\right)=O\left(d^{k-1}\right)$.

(v) If $k-1>s$ for any fixed $s>0$ then $\left\{\left|t^{*}\right|^{s}, 0<d \leq d_{0}\right\}$ is uniformly integrable. 
(vi) If $k>3$ then $E(t)=n^{*}+\nu-l-2+o(1)$.

From Theorem 2 of Chow et al. (1979), together with Lemma 4.1 (iii), we get

LEMMA 4.2. For any $s>0,\left\{|T|^{s}, 0<d \leq d_{0}\right\}$ is uniformly integrable.

LEMMA 4.3. If $k-1 \geq 2 s$ for any fixed $s>0$ then $E\left(\eta^{-s}\right)=O(1)$.

PROOF. Since $\eta \geq\left(n^{*}\right)^{-1}$ and $k-1-2 s \geq 0$, by Lemma 4.1 (iv) and (2.8) we have

$$
\begin{aligned}
E\left(\eta^{-s}\right) & =E\left\{\eta^{-s} I\left(\eta \leq \frac{1}{2}\right)\right\}+E\left\{\eta^{-s} I\left(\eta>\frac{1}{2}\right)\right\} \\
& \leq\left(n^{*}\right)^{s} P\left(\eta \leq \frac{1}{2}\right)+2^{s}=O\left(d^{k-1-2 s}\right)+2^{s}=O(1)
\end{aligned}
$$

where $I(A)$ denotes the indicator function of $A$. Thus the proof is complete.

LEMMA 4.4. Let $\xi$ be a random variable lying between 0 and 1 . If $k-1$ $>2 s$ for any fixed $s>1$ then

$$
\sup _{d>0} E\left\{\left|\bar{Y}_{t}\right|^{-s}\right\}<\infty \quad \text { and } \sup _{d>0} E\left\{\left|1+\xi\left(\bar{Y}_{t}-1\right)\right|^{-s}\right\}<\infty
$$

PROOF. Let $\mathfrak{F}_{n}$ be the $\sigma$-algebra generated by $\left\{\bar{Y}_{n}, \bar{Y}_{n+1}, \cdots\right\}$. Then it is well known that $\left\{\bar{Y}_{n}, \mathfrak{F}_{n}, n \geq k-1\right\}$ is a reversed martingale. Since $n \bar{Y}_{n}$ has the same distribution as $\chi_{n}^{2}$, it is easy to see that for any $p>-n / 2$

$$
E\left\{\left(\bar{Y}_{n}\right)^{p}\right\}=(2 / n)^{p}(\Gamma(p+n / 2) / \Gamma(n / 2)) .
$$

Hence by Doob's maximal inequality (e.g., Chow and Teicher (1988), p. 247) and (4.4) we get

$$
E\left\{\left|\bar{Y}_{t}\right|^{-s}\right\} \leq(s /(s-1))^{s} E\left\{\left(\bar{Y}_{k-1}\right)^{-s}\right\}<\infty
$$

for $k-1>2 s$, which gives the first assertion of the lemma since the right-hand side of (4.5) does not depend on $d$. It follows from (4.5) and the convexity of the function $x^{-s}$ on $(0, \infty)$ that

$$
\sup _{d>0} E\left\{\left|1+\xi\left(\bar{Y}_{t}-1\right)\right|^{-s}\right\} \leq 1+\sup _{d>0} E\left\{\left|\bar{Y}_{t}\right|^{-s}\right\}<\infty,
$$

which shows the second assertion. Therefore the proof of the lemma is complete.

We are now in the position to prove the results in Section 2.

PROOF of LEMMA 2.1. It is clear that

$$
s_{N}-\sigma=\frac{1}{2 \sigma}\left\{\left(s_{N}^{2}-\sigma^{2}\right)-\left(s_{N}-\sigma\right)^{2}\right\}
$$

Let

$$
Q=\eta t\left(l_{t}-1\right)-r
$$

Then it follows from (4.3) and (4.7) that 


$$
R=(\eta-1) t+Q .
$$

Since $E\left(S_{t}\right)=E(t)$ by Wald's lemma, we have, by (4.2) and (4.3),

$$
\begin{aligned}
\frac{1}{2 \sigma} E\left(s_{N}^{2}-\sigma^{2}\right) & =\frac{\sigma}{2} E\left\{\left(t^{-1}-\left(n^{*}\right)^{-1}\right) R\right\} \\
& =-\frac{\sigma}{2}\left(n^{*}\right)^{-1} E\left\{\eta^{-1}(\eta-1) R\right\} .
\end{aligned}
$$

From (4.3) and (4.8) we get

$$
\eta^{-1}(\eta-1) R=\left(t^{*}\right)^{2}+\eta^{-1}(\eta-1) Q .
$$

It follows from (2.10) and Lemma 4.1 that

$$
Q \stackrel{L}{\longrightarrow} l-H
$$

and $\left(t^{*}\right)^{2} \stackrel{L}{\longrightarrow} 2 \chi_{1}^{2}$, which, together with $(4.10)$, yield

$$
\eta^{-1}(\eta-1) R \stackrel{L}{\longrightarrow} 2 \chi_{1}^{2} \text {. }
$$

By (4.3), Hölder's inequality and Lemmas $4.1-4.3$ we have

$$
\begin{aligned}
& E\left\{\left|\eta^{-1}(\eta-1) R\right|^{3 / 2}\right\}=E\left\{\left|\eta^{-1} t^{*} T\right|^{3 / 2}\right\} \\
& \quad \leq\left\{E\left(\eta^{-3}\right)\right\}^{1 / 2}\left\{E\left(\left|t^{*}\right|^{6}\right)\right\}^{1 / 4}\left\{E\left(|T|^{6}\right)\right\}^{1 / 4}=O(1),
\end{aligned}
$$

which implies the uniform integrability of $\left\{\eta^{-1}(\eta-1) R, 0<d \leq d_{0}\right\}$. Hence from (4.9) and (4.12) we get

$$
\frac{1}{2 \sigma} E\left(s_{N}^{2}-\sigma^{2}\right)=-\sigma\left(n^{*}\right)^{-1}+o\left(d^{2}\right) .
$$

By Taylor's expansion and (4.2) we have

$$
s_{N}-\sigma=\frac{1}{2}\left\{\sigma^{2}+\xi\left(s_{N}^{2}-\sigma^{2}\right)\right\}^{-1 / 2}\left(s_{N}^{2}-\sigma^{2}\right)=\frac{\sigma}{2} B_{t}^{-1 / 2}\left(\bar{Y}_{t}-1\right),
$$

where $\xi$ is a random variable lying between 0 and 1 and $B_{t} \equiv 1+\xi\left(\bar{Y}_{t}-1\right)$. Thus we get $\left(s_{N}-\sigma\right)^{2}=\left(\sigma^{2} / 4\right) B_{t}^{-1}\left(\bar{Y}_{t}-1\right)^{2}$, which, together with (4.3), implies

$$
-\frac{1}{2 \sigma} E\left(s_{N}-\sigma\right)^{2}=-\frac{\sigma}{8 n^{*}} E\left(\eta^{-1} t^{-1} R^{2} B_{t}^{-1}\right) .
$$

In view of Anscombe's theorem (e.g., Corollary 1.4 of Woodroofe (1982)) we have

$$
R / \sqrt{2 t} \stackrel{L}{\longrightarrow} N(0,1),
$$

which, together with Lemma 4.1, yields

$$
\eta^{-1} t^{-1} R^{2} B_{t}^{-1} \stackrel{L}{\longrightarrow} 2 \chi_{1}^{2} \text {. }
$$

Choose $\alpha>1$ and $q>1$ such that $k-1>6 \alpha q$ and $p^{-1}+q^{-1}=1$. By (4.3), Hölder's inequality and Lemmas $4.2-4.4$ we get 


$$
\begin{aligned}
& E\left\{\left|\eta^{-1} t^{-1} R^{2} B_{t}^{-1}\right|^{\alpha}\right\}=E\left\{\left|\eta^{-2} T^{2} B_{t}^{-1}\right|^{\alpha}\right\} \\
& \quad \leq\left\{E\left(|T|^{2 \alpha p}\right)\right\}^{1 / p}\left\{E\left(\eta^{-3 \alpha q}\right)\right\}^{2 / 3 q}\left\{E\left(\left|B_{t}\right|^{-3 \alpha q}\right)\right\}^{1 / 3 q}=O(1),
\end{aligned}
$$

which implies the uniform integrability of $\left\{\eta^{-1} t^{-1} R^{2} B_{t}^{-1}, 0<d \leq d_{0}\right\}$. Hence (4.16) gives $E\left(\eta^{-1} t^{-1} R^{2} B_{t}^{-1}\right)=2+o(1)$, which, together with (4.14), yields

$$
-\frac{1}{2 \sigma} E\left(s_{N}-\sigma\right)^{2}=-\frac{\sigma}{4}\left(n^{*}\right)^{-1}+o\left(d^{2}\right) .
$$

Thus from (4.6), (4.13) and (4.17) we obtain the lemma. This completes the proof of Lemma 2.1 .

PROOF of PROPOSITION 2.1. Since $\bar{X}_{n}$ and $\left(s_{2}, \cdots, s_{n}\right)$ are independent, $\bar{X}_{n}$ and $I(N=n)$ are independent, given $N=n$. Thus

$$
E\left(\bar{X}_{N}\right)=\sum_{n=k}^{\infty} E\left(\bar{X}_{n} \mid N=n\right) P(N=n)=\mu .
$$

From this result, (2.11), and Lemma 2.1, we obtain the proposition. This completes the proof of Proposition 2.1.

We need the following lemma in order to prove Proposition 2.2.

LEMMA 4.5. If $k>6$ then $E\left(s_{N}^{-1}\right)=\sigma^{-1}+o(d)$.

Since the proof of Lemma 4.5 is similar to that of Lemma 2.1, we omit it.

PROOF of PROPOSITION 2.2. Proposition 2.2 is an immediate consequence of (2.8), (2.12), Proposition 2.1 and Lemma 4.5.

PROOF of THEOREM 2.1. For $n \geq 2$ let

$$
\begin{aligned}
& A_{n}=\left(n^{*} / n\right)^{1 / 2}, \quad B=a(2 / m)^{1 / 2}, \\
& C=b / \sqrt{m}, \quad C_{n}=\left\{2 b \sigma /\left(\left(s_{n}+\sigma\right) \sqrt{m}\right)\right\}(n /(n-1))^{1 / 2} \\
& D^{(1)}=0 \quad \text { and } \quad D^{(2)}=D_{n}^{(2)}=\left\{5 b d /\left(2 m z s_{n}\right)\right\}\left(n / n^{*}\right)^{1 / 2}
\end{aligned}
$$

From (2.5), (2.8) and (2.12) we have that for $j=1,2$

$$
z d^{-1}\left(\hat{\theta}_{N}^{(j)}-\theta\right)=A_{N}\left(B U_{N}+C_{N} V_{N}+D^{(j)}\right),
$$

which, together with (2.13), yields that for $j=1,2$

$$
P\left\{\theta \in I_{N}^{(j)}\right\}=P\left\{-z \leq A_{N}\left(B U_{N}+C_{N} V_{N}+D^{(j)}\right) \leq z\right\} .
$$

It follows from (2.6) that

(4.19) $V_{n} \stackrel{L}{\longrightarrow} V$ and $B U_{n}+C V_{n} \stackrel{L}{\longrightarrow} B U+C V$ as $n \rightarrow \infty$.

From Example 1.8 and Lemma 1.4 of Woodroofe (1982), $\left\{V_{n}, n \geq 2\right\}$ and $\left\{B U_{n}\right.$ $\left.+C V_{n}, n \geq 2\right\}$ are uniformly continuous in probability. Hence by (4.19) and Anscombe's theorem (e.g., Theorem 1.4 of Woodroofe (1982)) we have

(4.20) $V_{N} \stackrel{L}{\longrightarrow} V$ and $B U_{N}+C V_{N} \stackrel{L}{\longrightarrow} B U+C V$ as $d \rightarrow 0$.

Since $N \stackrel{\text { a.s. }}{\longrightarrow} \infty, N / n^{*} \stackrel{\text { a.s. }}{\longrightarrow} 1$ and $s_{N} \stackrel{\text { a.s. }}{\longrightarrow} \sigma$ as $d \rightarrow 0$, it follows from (4.20) that for $j=1,2$ 


$$
\begin{aligned}
& \left|\left(B U_{N}+C_{N} V_{N}+D^{(j)}\right)-\left(B U_{N}+C V_{N}\right)\right| \\
& \quad=\left|\left(C_{N}-C\right) V_{N}+D^{(j)}\right| \stackrel{p}{\longrightarrow} 0 \text { as } d \rightarrow 0,
\end{aligned}
$$

which, together with (4.20), implies that for $j=1,2$

$$
B U_{N}+C_{N} V_{N}+D^{(j)} \stackrel{L}{\longrightarrow} B U+C V \text { as } d \rightarrow 0 .
$$

Thus, by the fact that $A_{N} \stackrel{\text { a.s. }}{\longrightarrow} 1$ as $d \rightarrow 0$ we get that for $j=1,2$

$$
A_{N}\left(B U_{N}+C_{N} V_{N}+D^{(j)}\right) \stackrel{L}{\longrightarrow} B U+C V \text { as } d \rightarrow 0,
$$

which, together with (4.18) and the fact that $B U+C V$ has a standard normal distribution, yields that for $j=1,2$

$$
P\left\{\theta \in I_{N}^{(j)}\right\} \rightarrow \Phi(z)-\Phi(-z)=1-\alpha \text { as } d \rightarrow 0 .
$$

Therefore the proof of Theorem 2.1 is complete.

PROOF of THEOREM 2.2. This theorem is an immediate consequence of (4.2), Lemma 4.1 (vi) and the fact that $l=1-L$.

Finally, we shall give the outline of the proof of Theorem 3.1. Before that, we state a lemma without proof.

LEMMA 4.6. The following results hold.

(i) $E\left(\bar{Y}_{t}-1\right)^{2}=\frac{2}{n^{*}}+(-2 \nu+2 l+16)\left(n^{*}\right)^{-2}+o\left(d^{4}\right)$ if $k>7$.

(ii) $E\left(\bar{Y}_{t}-1\right)^{3}=-16\left(n^{*}\right)^{-2}+o\left(d^{4}\right)$ if $k>8$.

(iii) $E\left[\left(\bar{Y}_{t}-1\right)^{4} B_{t}^{-7 / 2}\right]=12\left(n^{*}\right)^{-2}+o\left(d^{4}\right)$ if $k>16$, where $B_{t} \equiv 1+\xi\left(\bar{Y}_{t}-1\right)$ and $\xi$ is a random variable lying between 0 and 1 .

(iv) $E\left(s_{N}^{-2}\right)=\sigma^{-2}+o(1)$ if $k>6$.

(v) $E\left(1-\sigma s_{N}^{-1}\right)=-\frac{7}{4 n^{*}}+o\left(d^{2}\right)$ if $k>10$.

PROOF of THEOREM 3.1. Since $\bar{X}_{n}$ and $\left(s_{2}^{2}, \cdots, s_{n}^{2}\right)$ are independent, it follows from the definition of $N$ that

$$
E\left\{\hat{\theta}_{N}^{(1)}-\theta\right\}^{2}=a^{2} \sigma^{2} E\left(N^{-1}\right)+b^{2} E\left(s_{N}-\sigma\right)^{2} .
$$

From Taylor's expansion, (4.2), (4.3), Theorem 2.2 and Lemma 4.6 we have

(4.22) $E\left(s_{N}-\sigma\right)^{2}$

$$
\begin{aligned}
& =\left(\sigma^{2} / 4\right) E\left(\bar{Y}_{t}-1\right)^{2}-\left(\sigma^{2} / 8\right) E\left(\bar{Y}_{t}-1\right)^{3}+\left(5 \sigma^{2} / 64\right) E\left[\left(\bar{Y}_{t}-1\right)^{4} B_{t}^{-7 / 2}\right] \\
& =\frac{\sigma^{2}}{2 n^{*}}+\frac{\sigma^{2}}{\left(n^{*}\right)^{2}}\left(-\frac{\nu}{2}+\frac{l}{2}+\frac{111}{16}\right)+o\left(d^{4}\right)
\end{aligned}
$$

and

(4.23) $E\left(N^{-1}\right)=\left(n^{*}\right)^{-1}-\left(n^{*}\right)^{-2}(\nu-l-1+o(1))$

$$
+\left(n^{*}\right)^{-2} E\left[\left(t^{*}+\left(n^{*}\right)^{-1 / 2}\right)^{2}\left\{1+\xi\left(\eta-1+\left(n^{*}\right)^{-1}\right)\right\}^{-3}\right],
$$

where $\xi$ is a random variable with $\xi \in(0,1)$. From Lemma 4.1 we have 


$$
J \equiv\left(t^{*}+\left(n^{*}\right)^{-1 / 2}\right)^{2}\left\{1+\xi\left(\eta-1+\left(n^{*}\right)^{-1}\right)\right\}^{-3} \stackrel{L}{\longrightarrow} 2 \chi_{1}^{2},
$$

which, together with the uniform integrability of $\left\{J, 0<d \leq d_{0}\right\}$ and (4.23), implies

$$
E\left(N^{-1}\right)=\left(n^{*}\right)^{-1}+(-\nu+l+3)\left(n^{*}\right)^{-2}+o\left(d^{4}\right) .
$$

From (4.21), (4.22), (4.24) and the fact that $l=1-L$ we obtain (3.1). It follows from (2.8) and (2.12) that

$$
E\left\{\hat{\theta}_{N}^{(2)}-\theta\right\}^{2}=E\left\{\hat{\theta}_{N}^{(1)}-\theta\right\}^{2}+\frac{5 b^{2} \sigma^{2}}{2 n^{*}} E\left(1-\sigma s_{N}^{-1}\right)+\frac{25 b^{2} \sigma^{4}}{16\left(n^{*}\right)^{2}} E\left(s_{N}^{-2}\right),
$$

which, together with Lemma 4.6 and (3.1), yields (3.2). Therefore the proof of Theorem 3.1 is complete.

\section{Acknowledgements}

The second author (E.I.) has been partially supported by a Grant-in-Aid for Science Research, number 09640251, from the Ministry of Education, Science and Culture, Japan. The authors would like to thank the referees for their useful comments.

\section{REFERENCES}

[1] Billingsley, P. (1968). Convergence of Probability Measures, Wiley, New York.

[2] Callaert, H. and Janssen, P. (1981). The convergence rate of fixed-width sequential confidence intervals for the mean, Sankhyā Ser. A., 43, 211-219.

[ 3 ] Chow, Y. S., Hsiung, C. A. and Lai, T. L. (1979). Extended renewal theory and moment convergence in Anscombe's theorem, Ann. Probab., 7, 304-318.

[4] Chow, Y. S. and Robbins, H. (1965). On the asymptotic theory of fixed-width sequential confidence intervals for the mean, Ann. Math. Statist., 36, 457-462.

[5] Chow, Y. S., Robbins, H. and Teicher, H. (1965). Moments of randomly stopped sums, Ann. Math. Statist., 36, 789-799.

[6] Chow, Y. S. and Teicher, H. (1988). Probability Theory: Independence, Interchangeability and Martingales, Second Edition, Springer-Verlag, New York.

[7] Ghosh, M. and Mukhopadhyay, N. (1989). Sequential estimation of the percentiles of exponential and normal distributions, South African Statist. J., 23, 251-268.

[8] Hirose, K., Isogai, E. and Uno, C. (1997). The convergence rate of fixed-width sequential confidence intervals for a parameter of an exponential distribution, Ann. Inst. Statist. Math., 49, 199-209.

[9] Isogai, E., Hirose, K. and Uno, C. (1995). Fixed-width confidence interval estimation of a parameter of an exponential distribution, submitted for publication.

[10] Jurečková, J. (1991). Confidence sets and intervals, In Handbook of Sequential Analysis, (Ghosh, B. K. and Sen, P. K. eds.), Chapter 11, 269-281, Marcel Dekker, Inc., New York.

[11] Mukhopadhyay, N. and Datta, S. (1996). On sequential fixed-width confidence intervals for the mean and second-order expansions of the associated coverage probabilities, Ann. Inst. Statist. Math., 48, 497-507.

[12] Woodroofe, M. (1977). Second order approximations for sequential point and interval estimation, Ann. Statist., 5, 984-995.

[13] Woodroofe, M. (1982). Nonlinear Renewal Theory in Sequential Analysis, CBMS Monograph No. 39, Soc. Indust. Appl. Math., Philadelphia. 